\title{
Evaluation of Second-Trimester Maternal Serum AFP Value in the Detection of Congenital Heart Defects
}

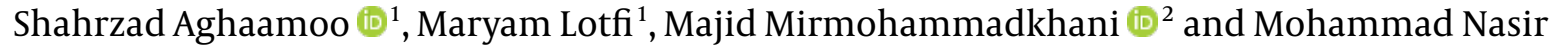 \\ Hemmatian (iD) $3,4,{ }^{*}$ \\ ${ }^{1}$ Abnormal Uterine Bleeding Research Center, Semnan University of Medical Sciences, Semnan, Iran \\ ${ }^{2}$ Social Determinants of Health Research Center, Faculty of Medicine, Semnan University of Medical Sciences, Semnan, Iran \\ ${ }^{3}$ Department of Pediatrics, Semnan University of Medical Sciences, Semnan, Iran \\ ${ }^{4}$ Yas Hospital Complex, Tehran University of Medical Sciences (TUMS), Tehran, Iran \\ "Corresponding author: Yas Hospital Complex , Tehran University of Medical Sciences (TUMS), Tehran, Iran. Email: nasir.hematian90@yahoo.com
}

Received 2020 December 06; Revised 2021 October 10; Accepted 2021 October 11.

\begin{abstract}
Background: Congenital heart diseases, as the most common congenital anomaly, are a major cause of serious morbidity and mortality.

Objectives: This study aimed to evaluate the level of alpha-fetoprotein in the second trimester of pregnancy and its association with congenital heart defects.

Methods: Following a cross-sectional design, singleton pregnant women in the second-trimester were enrolled for the $\alpha$ fetoprotein (AFP) screening test. Those with normal screening results at the first and second tests were assigned to the control group. Mothers with nuchal translucency (NT) screening equal or greater than $3 \%$ of the 99th percentile, without chromosomal anomalies, confirmed with amniocentesis, were referred for a fetal echocardiogram and in presence of congenital heart defects symptoms, assigned to the intervention group. The study groups were compared concerning the serum AFP level and maternal and neonatal outcomes in the second trimester of pregnancy. Statistical significance was considered when P-value $<0.05$.

Results: A total of 270 women were enrolled in the study, with a mean AFP level of $1.868 \pm 0.87$ and $1.374 \pm 0.39$ in the case and control groups, respectively. The mean level of AFP was significantly different between the two groups $(\mathrm{P}<0.001)$. In the second trimester, an AFP level lower than 1 was associated with a significant increase in the likelihood of developing congenital heart anomalies in the fetus.

Conclusions: This study demonstrated that the value of maternal AFP level in the second trimester can be considered as an appropriate screening test to predict the incidence of congenital heart defects in neonates.
\end{abstract}

Keywords: Congenital Heart Defect, Prenatal Screening, Alpha-Fetoprotein

\section{Background}

Congenital heart defects (CHD) are the most common congenital anomalies, with an estimated incidence of 8 per 1,000 live births in the United States (1). Severe cases of CHD often require major cardiac surgeries, which may result in death (2). In addition, even a successful surgery is associated with a wide spectrum of adverse outcomes, which lead to a life expectancy drop (3-5).

Hence, early diagnosis and in-time intervention are necessary to reduce both mortality and morbidity of the disease $(1,2)$. Furthermore, early diagnosis has a major contribution to reducing healthcare expenditures (1-7). The first fetal echocardiography was performed in Denmark in 1995 to identify CHD at 18 to 21 weeks of gestation. Although initially echocardiography was considered as a screening tool to identify fetal heart defects, due to its inability to observe the four-chamber and three-vessel views, its administration was limited; so that, at the best condition, the diagnostic power of echocardiography was only 50\% (8).

Nuchal translucency (NT), which must be done between 11 and 14 weeks of pregnancy, significantly increased the diagnostic power of detecting CHE so that the power of simultaneous NT and echocardiography is up to $90 \%$ (9, 10). However, echocardiography not only is difficult and expensive but also requires advanced equipment, which have translated into limited access to this service. Therefore, there is a need for an inexpensive, accurate screening tool that is available anywhere (2,11-13). Some studies have investigated the value of maternal serum biomarker levels in the second trimester for the early diagnosis of CHD. However, previous studies could not reach a definite 
conclusion, and their results are not generalizable to the entire population $(14,15)$. Elevated NT measurements $(16)$ and abnormal levels of serum biomarkers in the second trimester are associated with CHD (17). Human chorionic gonadotropin, $\alpha$-fetoprotein (AFP), non-conjugated estradiol, and inhibin are routine screening tests at the second trimester $(7,18)$.

Only a few studies have been recently conducted on CHD diagnostic tools, and there is no sufficient knowledge for introducing the best screening and diagnostic method for CHD during pregnancy as a basis for designing and implementing targeted interventions for early diagnosis and prevention. Fetal echocardiography not only is expensive but also is not accessible and affordable for all pregnant women, which indicates the need for an inexpensive and available tool for early screening of CHD. The AFP measurement is widely used in the second trimester and may be useful in the early detection of CHD. By this time, a few studies evaluated the association between the findings of screening tests in the second trimester and CHDs. In addition, most of them focused on the screening tests in the first trimester, especially on NT, or analyzed previously recorded data, and less attention has been paid to the fetal and neonatal outcomes, as well as the results of maternal examinations $(1,15)$.

\section{Objectives}

This study aimed to evaluate the association between the level of AFP in the second trimester and congenital heart defects.

\section{Methods}

\subsection{Patients}

Following a cross-sectional design, 270 singleton pregnant women (90 in the intervention group and 180 in the control group) aged 18 to 40 years in the second trimester with normal and low-risk pregnancy referred to obstetrics clinic from March 2015 to February 2017 enrolled in the study. Participants were selected using easy sampling among eligible cases. By considering a 95\% confidence interval and 5\% error, the sample size was estimated using the equation for minimum sample size. The inclusion criteria were having a low-risk pregnancy, being aged 18 to 40 years, singleton pregnancy, body mass index (BMI) of $<25$. All cases with systemic diseases such as diabetes, lupus, collagen vascular disorders, hypertension and phenotypic abnormalities in neonates after birth, past history of chromosomal abnormalities, abnormal karyotype, therapy for infertility, and use of anticonvulsants agent, and alcohol and drug abuse were excluded.

\subsection{Experimental Design}

Maternal demographic data, history, and clinical and para-clinical findings were recorded. Echocardiography was performed in the second trimester, once after the birth, by a pediatric cardiologist. CHD was defined according to the International Classification of Disease, Tenth Revision (ICD-10-CM), which is atrial or ventricular dysfunction and anomalies in the aorta and pulmonary artery (19).

Second-trimester prenatal screening (AFP measurement) was performed at 14 to 17 weeks of gestational. All cases with normal results for NT and prenatal screening tests at first and second trimesters were assigned to the control group. Mothers with abnormal screening findings at the 18 - 22 weeks of gestational, as well as mothers with NT criteria equal and higher than 3\% or higher than 99th percentile at 11 - 13 weeks of gestational, were referred for amniocentesis. In the absence of chromosomal anomaly, fetal echocardiography was performed, and those with CHD were assigned to the intervention group.

\subsection{Ethical Considerations}

All experimental protocols have been conducted in compliance with the regulations of the Research Ethics Committee of the University and the Iranian Ethical Guidelines in Research. Also, the research purpose and methodology were subjected to scrutiny by the Ethics Committee of Semnan University of Medical Sciences (IR.SEMUMS.REC.1396.25). In addition, written informed consent was obtained from all participants before entering the study and after a comprehensive introduction to the study protocol.

\subsection{Statistical Analysis}

The level of AFP in the second trimester was compared based on maternal and neonatal parameters for both groups. The Kolmogorov-Smirnov test was applied to test for a normal distribution. Data analysis was administered by $t$-test, Mann-Whitney U, chi-square, and Fisher's exact test using SPSS version 21. Statistical significance was considered when P-value $<0.05$.

\section{Results}

A total of 270 eligible pregnant women were enrolled in this study, of whom 9 were excluded due to delivery in another city, refusal to continue the study, and presence of postnatal anomalies, such as clubbing and cleft lip. Finally, 261 patients were assigned in each of the study groups, 90 cases, and 171 controls. Echocardiography was performed between 18 - 22 weeks of gestational age (mean \pm SD, 21.99 $\pm 4.6)$ and then, if necessary, repeated until 37 weeks of gestation. AFP test was performed between 14 to 20 weeks of gestational age (mean $\pm S D, 15.66 \pm 0.8$ ). 
The mean age of participants was $28.23 \pm 6.6$ years (controls: $27.82 \pm 5.5$ and cases: $28.77 \pm 4.5$ years). The age distribution was normal in both groups $(\mathrm{P}=0.106, \mathrm{Z}=1.212)$. The incidence of neonatal complications in the control and intervention groups was $29.2 \%$ (50 infants) and $44.4 \%$ (40 infants), respectively. The most common neonatal complication was respiratory problems (Table 1 ). The most common maternal complication was preeclampsia (20 cases), followed by postpartum hemorrhage (16 cases). In the control group, the most common complication was postpartum hemorrhage (23 cases), followed by preeclampsia (19 cases). Fifteen infants in the case group were admitted to the neonatal intensive care unit (NICU), of whom five died. In the control group, 12 infants were admitted to the NICU, whom all survived. Out of 90 cases with congenital heart defects, 12 were critical (Table 2). The mean level of AFP was $1.868 \pm 0.86$ and $1.374 \pm 0.39$ in cases and controls, respectively. The mean level of AFP was significantly different between the two groups $(\mathrm{P}<0.001)$.

The mean level of AFP was significantly different in mothers with three or more parities but not significant in nulliparous and uniparous women. In mothers with three or more parities, alpha-fetoprotein levels were significantly reduced, which can be attributed to congenital anomalies (Table 3). AFP level was significantly different in mothers whose infants had critical and noncritical cardiac defects $(P=0.001)$. There was a significant difference in the level of AFP in mothers of healthy infants and those with non-critical cardiac defects compared to mothers of infants with CHDs $(\mathrm{P}<0.001)$ (Table 4$)$. The mean level of AFP in mothers of complicated and uncomplicated infants was significantly higher in the intervention group than in the control group $(\mathrm{P}=0.044)$. The mean level of AFP in mothers of uncomplicated infants was not significantly different in the control group compared to the intervention group $(P=0.070)$. The mean level of AFP in mothers of infants admitted to ICU was higher in controls than in cases $(\mathrm{P}<0.001)$. There was a significant difference between the study groups concerning the AFP levels in mothers of infants admitted to NICU $(P=0.016)$. Five infants in the intervention group died, and there was no neonatal death in the control group. A cut-off point equal to "1" was considered for alfafitoprotein level, and all subjects in both groups were evaluated in one set for the incidence of heart defects in the fetus. The frequency of CHDs in mothers with an alfafitoprotein protein level lower than "1" was increased by 11-fold (Exp. $\mathrm{B}=11.081, \mathrm{P}<0.001$ ).

\section{Discussion}

This study compared the AFP level in the second trimester in mothers of neonates with and without congenital heart defects (CHDs) based on fetal echocardio- graphy in the second trimester. So, AFP level was measured in the second trimester and mothers underwent fetal echocardiography.

CHD was diagnosed in fetuses of 90 pregnant women (171 were healthy). According to the findings, AFP level was lower in mothers of neonates with $\mathrm{CHD}(\mathrm{P}=0.004)$. The level of AFP was not associated with the mother's age and gestational age. In women with 3 parity and more, there was a significant difference regarding the mean level of AFP in cases with and without $\mathrm{CHD}(\mathrm{P}=0.003)$. There was a significant difference concerning the mean level of AFP in mothers of a neonate with critical and noncritical CHD ( $P>0.001)$, and between mothers of the neonate with critical CHD and without CHD ( $\mathrm{P}<0.001)$. The level of AFP in women with and without maternal and neonatal complications was also significant $(\mathrm{P}=0.044)$. In mothers of an infant with CHD admitted to NICU, AFP level was lower than that of mothers of an infant without CHD admitted to NICU $(\mathrm{P}<0.001)$. At the second trimester, an AFP level lower than 1 was associated with an increased chance of (by 11-fold) having an infant with CHD (Exp. B = 11.081, P < 0.001).

Few studies have investigated the results of screening tests in the second trimester and their association with congenital heart diseases. For instance, Borelli et al. investigated the association between the risk of CHDs with serum biomarkers levels that are routinely measured in the first and second trimesters. They studied 118194 pregnant women and reported that 284 newborns with CHD were born (1). In mothers with $\beta$-HCG below the 10 th percentile, the chance of having a neonate with CHDs was 1.6 times lower than that of others; in cases with an NT higher than the 95th percentile, the chance of having a neonate with congenital heart disease was 2 times more than others. Among those with CHD-related risk factors, only $21.8 \%$ were suffering from the disease; therefore, the presence of risk factors alone has no sensitivity and specificity in early diagnosis of CHD; thus, concerning the significant association between serum biomarkers and CHD, measurement of these biomarkers and their association with CHD is necessary, even in women without any risk factors (1).

We evaluated the level of AFP in mothers with and without an infant suffering from congenital heart disease, and a significant difference was found between the study groups. In addition, other maternal factors did not play a role in the incidence of CHDs.

In this study, there was a significant difference between AFP levels in mothers with at least 3 parities; however, it was not significant in mothers with parities less than 3 . In addition, the incidence of congenital heart disease in infants of mothers with AFP levels of more than 1 was higher by 11 -folds.

Alves Rocha et al. reviewed the articles that inves- 


\begin{tabular}{|c|c|c|c|c|c|}
\hline \multirow{2}{*}{ Neonatal Outcomes } & \multicolumn{2}{|c|}{ Case } & \multicolumn{2}{|c|}{ Control } & \multirow{2}{*}{ Total } \\
\hline & No. & $\%$ & No. & $\%$ & \\
\hline Meconium aspiration & 5 & 12.5 & 9 & 18.0 & 14 \\
\hline Prematurity & 7 & 17.5 & 14 & 28.0 & 21 \\
\hline Cyanosis & 12 & 30.0 & 1 & 2.0 & 13 \\
\hline Others & 4 & 10.0 & 10 & 20.0 & 14 \\
\hline
\end{tabular}

\begin{tabular}{|lcc}
\hline Table 2. Frequency of Critical and Non-critical Congenital Heart Defects & \\
\hline Type of Congenital Heart Defects & $\%$ & No. \\
\hline Critical & & \\
\hline Tetralogy of Fallot & 2.2 & 2 \\
\hline d-Transposition of Great Arteries & 3.3 & 3 \\
\hline Hypo plastic left ventricle & 2.2 & 2 \\
\hline Common arterial trunk & 1.1 & 1 \\
\hline Tricuspid atresia & 2.2 & 2 \\
\hline Aortic atresia & 1.1 & 1 \\
\hline Atresia of pulmonary artery & 1.1 & 1 \\
\hline Non critical & & \\
\hline Ventricular septal defect (VSD) & 23.3 & 21 \\
\hline Atrial septal defect & 18.8 & 17 \\
\hline Congenital heart block & 10.0 & 9 \\
\hline Dextrocardia & 3.3 & 3 \\
\hline Coarctation of aorta & 3.3 & 3 \\
\hline Atrioventricular septal defect(AVSD)21.1 & 6.7 & 6 \\
\hline Other & 21.1 & 19 \\
\hline
\end{tabular}

tigated the screening of congenital heart disease in the second trimester. They reported that although various tools are available for screening of CHD such as echocardiography, ultrasound, and color Doppler, but, due to the high cost, these methods are not routinely performed and sometimes are ignored, especially in mothers without risk factors for CHD like diabetes. Therefore, it is necessary to use an inexpensive and accessible tool for CHD screening. The authors point out that ultrasound and fetal echocardiography should be used as a means for confirmation of CHD and other screening methods. They consider the second trimester as the best time for screening and diagnosis of fetal heart defects (20).

In our study, screening tests (AFP levels) were performed in the second trimester, and complementary diagnostic techniques were conducted in the third trimester. The results of our study indicated that the mean level of
AFP was lower in mothers of an infant with CHD, with due attention to an 11-fold increase in the incidence of CHD in mothers with AFP level less than 1 in the second trimester, this test is suitable for CHD screening. Jelliffe-Pawlowski et al. evaluated the prediction power of beta-human chorionic gonadotropin ( $\beta$-HCG), AFP, and unconjugated estriol (uE3) in the second trimester for CHD in 19402 pregnant women without chromosomal abnormalities in the fetus. The results showed that the measurement of biomarkers in the second trimester can be used as a useful tool for the early diagnosis of CHD in cases without chromosomal abnormalities (6). In the same vein, Jelliffe-Pawlowski et al. also reported similar results (6). In our study, an AFP level less than "1" was associated with an 11-fold increase in the risk of CHD in infants, and according to the results, it can be used as a maternal screening test to determine the probability of congenital heart disease in infants.

In another study, Jelliffe-Pawlowski et al. evaluated screening findings in the second trimester and the association between ultrasound findings and the fetal CHD and reported that $42.7 \%$ of all CHD cases were in mothers with a $\beta$-HCG level above the 95th percentile. In $92.9 \%$ of patients with both CHD and trisomy 18 , the $\beta$-HCG values were lower than the fifth percentile. This study suggests that, considering the association between CHD and chromosomal abnormalities in neonates, screening tests in the second trimester can be an effective tool for predicting and diagnosing neonates with CHD, especially in mothers without risk factors who are neglected for fetal heart defects (15). It should be noted that this study has applied a methodology similar to our research, but we evaluated the AFP level in the second trimester. In our study, the AFP level was less than 1 in $73.3 \%$ of mothers who had a neonate with CHD. Our findings also indicated that the AFP level could be considered as a proper biomarker for CHD screening. A few studies have evaluated the value of screening tests in the second trimester and their association with CHD (2123). The results of our study indicate the value of maternal AFP level in the second trimester as a screening test to predict the incidence of $\mathrm{CHD}$. Suard et al. reported that the diagnostic value of maternal screen tests in the diagnosis 


\begin{tabular}{|c|c|c|c|c|c|}
\hline \multirow{2}{*}{\multicolumn{2}{|c|}{ Parity }} & \multirow{2}{*}{ No. } & \multicolumn{2}{|c|}{$\alpha$-fetoprotein level } & \multirow{2}{*}{ P Value } \\
\hline & & & Mean & SD & \\
\hline \multirow[t]{3}{*}{1} & & & & & 0.099 \\
\hline & Case & 40 & 1.053 & 0.97 & \\
\hline & Control & 72 & 1.323 & 0.35 & \\
\hline \multirow[t]{3}{*}{2} & & & & & 0.206 \\
\hline & Case & 29 & 1.153 & 0.97 & \\
\hline & Control & 58 & 1.396 & 0.40 & \\
\hline \multirow[t]{3}{*}{$\geq 3$} & & & & & 0.003 \\
\hline & Case & 21 & 1.057 & 0.46 & \\
\hline & Control & 41 & 1.435 & 0.45 & \\
\hline \multirow{2}{*}{\multicolumn{2}{|c|}{ Groups/Heart Defects }} & No & \multicolumn{2}{|c|}{ Alpha-Fetoprotein Level } & DValne \\
\hline & & 100 & Mean & SD & Faiue \\
\hline \multirow[t]{3}{*}{ Case } & & & & & 0.001 \\
\hline & Critical & 12 & 0.668 & 0.29 & \\
\hline & Non critical & 78 & 1.150 & 0.91 & \\
\hline \multirow[t]{3}{*}{ Total } & & & & & $<0.001$ \\
\hline & Critical & 12 & 0.668 & 0.29 & \\
\hline & Non critical & 249 & 1.304 & 0.61 & \\
\hline
\end{tabular}

of congenital heart disease to be $71.5 \%$ (22).

\subsection{Conclusions}

This study intended to compare the maternal AFP level in the second trimester between mothers of neonates with and without congenital heart defects (CHD). Although a significant difference was observed, a prospective study with a larger study with more participants can better confirm the results of this study. The results showed that maternal AFP level in the second trimester is a proper screening test to predict the incidence of CHD in neonates. The maternal AFP level in the second trimester less than 1 is associated with an 11-fold increase in the incidence of CHD.

\section{Footnotes}

Authors' Contribution: Shahrzad Aghaamoo, Mohammad Nasir Hematian, and Maryam Lotfi prepared grant submissions for the project and was also involved in the planning, preparation, and approval of the original questionnaire. Maryam Lotfi conducted the questionnaire survey and data collection. Majid Mirmohammadkhani conducted statistical analysis. All authors contributed to the writing of the article, and read and approved the final manuscript.

Conflict of Interests: The authors declare that there is no conflict of interest.

Ethical Approval: This study was also approved and supported by the Ethics Committee of Semnan University of Medical Sciences (ethical code: IR.SEMUMS.REC.1396.25).
Funding/Support: This work was a project funded by the Council of Research, Semnan University of Medical Sciences (grant\#1224).

Informed Consent: All participants had informed consent.

\section{References}

1. Borelli M, Baer RJ, Chambers CD, Smith TC, Jelliffe-Pawlowski LL. Critical congenital heart defects and abnormal levels of routinely collected first- and second-trimester biomarkers. Am J Med Genet A. 2017;173(2):368-74. doi: 10.1002/ajmg.a.38013. [PubMed: 27739239].

2. Jorgensen DE, Vejlstrup N, Jorgensen C, Maroun LL, Steensberg J, Hessellund A, et al. Prenatal detection of congenital heart disease in a low risk population undergoing first and second trimester screening. Prenat Diagn. 2015;35(4):325-30. doi: 10.1002/pd.4525. [PubMed: 25352400].

3. Karsdorp PA, Everaerd W, Kindt M, Mulder BJ. Psychological and cognitive functioning in children and adolescents with congenital heart disease: A meta-analysis.J Pediatr Psychol. 2007;32(5):527-41. doi: 10.1093/jpepsy/jslo47. [PubMed: 17182669].

4. LüscherTF. Outcome of congenital heart disease with modern cardiac care. Eur Heart J. 2018;39(12):969-71. doi: 10.1093/eurheartj/ehy166.

5. Zomer AC, Vaartjes I, Grobbee DE, Mulder BJ. Adult congenital heart disease: New challenges. Int J Cardiol. 2013;163(2):105-7. doi: 10.1016/j.ijcard.2012.03.035. [PubMed: 22445874].

6. Jelliffe-Pawlowski LL, Walton-Haynes L, Currier RJ. Identification of second trimester screen positive pregnancies at increased risk for congenital heart defects. Prenat Diagn. 2009;29(6):570-7. doi: 10.1002/pd.2239. [PubMed: 19266536].

7. Cunningham FG, Bloom SL, Hauth JC, Rouse DJ, Spong CY. Normal Labor and Delivery. Williams Obstetrics. 23rd ed. New York, USA: McGraw Hill Co; 2010.

8. Zhang YF, Zeng XL, Zhao EF, Lu HW. Diagnostic Value of Fetal Echocardiography for Congenital Heart Disease: A Systematic Review and Meta-Analysis. Medicine (Baltimore). 2015;94(42). e1759. doi: 
10.1097/MD.0000000000001759. [PubMed: 26496297]. [PubMed Central: PMC4620824].

9. Ekelund CK, Jorgensen FS, Petersen OB, Sundberg K, Tabor A; Danish Fetal Medicine Research Group. Impact of a new national screening policy for Down's syndrome in Denmark: population based cohort study. BMJ. 2008;337. a2547. doi: 10.1136/bmj.a2547. [PubMed: 19039015]. [PubMed Central: PMC2590884].

10. Ekelund CK, Andersen HJ, Christensen J, Ersbak V, Farlie R, Henriques $\mathrm{C}$, et al. [Down's syndrome risk assessment in Denmark-secondary publication]. Ugeskr Laeger. 2010;172(23):1759-61. Danish. [PubMed: 20552746].

11. Pinto NM, Keenan HT, Minich LL, Puchalski MD, Heywood M, Botto LD. Barriers to prenatal detection of congenital heart disease: a population-based study. Ultrasound Obstet Gynecol. 2012;40(4):418-25. doi: 10.1002/uog.10116. [PubMed: 21998002].

12. Pinto NM, Nelson R, Puchalski M, Metz TD, Smith KJ. Cost-effectiveness of prenatal screening strategies for congenital heart disease. $\mathrm{Ul}$ trasound Obstet Gynecol. 2014;44(1):50-7. doi: 10.1002/uog.13287. [PubMed: 24357432]. [PubMed Central: PMC5278773].

13. Kondo M, Ohishi A, Baba T, Fujita T, Iijima S. Can echocardiographic screening in the early days of life detect critical congenital heart disease among apparently healthy newborns? BMC Pediatr. 2018;18(1):359. doi: 10.1186/s12887-018-1344-z. [PubMed: 30453920]. [PubMed Central: PMC6241044].

14. O'Leary P, Breheny N, Dickinson JE, Bower C, Goldblatt J, Hewitt $B$, et al. First-trimester combined screening for Down syndrome and other fetal anomalies. Obstet Gynecol. 2006;107(4):869-76. doi: 10.1097/01.AOG.0000207562.09858.16. [PubMed: 16582125].

15. Jelliffe-Pawlowski LL, Walton-Haynes L, Currier RJ. Using second trimester ultrasound and maternal serum biomarker data to help detect congenital heart defects in pregnancies with positive triplemarker screening results. Am J Med Genet A. 2008;146A(19):2455-67. doi:10.1002/ajmg.a.32513. [PubMed: 18785270].
16. Jelliffe-Pawlowski LL, Norton ME, Shaw GM, Baer RJ, Flessel MC, Goldman S, et al. Risk of critical congenital heart defects by nuchal translucency norms. Am J Obstet Gynecol. 2015;212(4):518 e1-10. doi: 10.1016/j.ajog.2014.10.1102. [PubMed: 25448520].

17. Hill GD, Block JR, Tanem JB, Frommelt MA. Disparities in the prenatal detection of critical congenital heart disease. Prenat Diagn. 2015;35(9):859-63. doi: 10.1002/pd.4622. [PubMed: 25989740]. [PubMed Central: PMC4558244]

18. Yazdani S, Rouholahnejad R, Asnafi N, Sharbatdaran M, Zakershob $\mathrm{M}$, Bouzari Z. Correlation of pregnancy outcome with quadruple screening test at second trimester. Med J Islam Repub Iran. 2015;29:281. [PubMed: 26913244]. [PubMed Central: PMC4764288].

19. Centers for Disease Control and Preventation. International Classification of Diseases,Ninth Revision, Clinical Modification (ICD-9-CM). USA Centers for Disease Control and Preventation; [cited 2021]. Available from: https://www.cdc.gov/nchs/icd/icd9cm.htm.

20. Alves Rocha L, Araujo Junior E, Rolo LC, Barros FS, Silva KP, Martinez $\mathrm{LH}$, et al. Screening of congenital heart disease in the second trimester of pregnancy: Current knowledge and new perspectives to the clinical practice. Cardiol Young. 2014;24(3):388-96. doi: 10.1017/S1047951113001558. [PubMed: 24229491].

21. Naghizadeh S, Azari S, Fathnejad Kazemi A, Ebrahimpour Mirzarezaei M, Alizadeh S. [Assessing and comparing result of amniocentesis and tripel marker tests to detection of Aneuploidies]. Nurs Midwifery J. 2015;13(7):596-604. Persian.

22. Suard C, Flori A, Paoli F, Loundou A, Fouilloux V, Sigaudy S, et al. Accuracy of prenatal screening for congenital heart disease in population: A retrospective study in Southern France. PLoS One. 2020;15(10) e0239476. doi: 10.1371/journal.pone.0239476. [PubMed: 33017437]. [PubMed Central: PMC7535055].

23. Pham A, Melchior M. Screening for fetal congenital heart disease. CMAJ. 2017;189(12). E468. doi: 10.1503/cmaj.160700. [PubMed: 28385862]. [PubMed Central: PMC5367993]. 